\title{
Visualizing the Spatial and Temporal Distribution of User Interaction Data Collected in Three-Dimensional Virtual Worlds
}

\author{
Katy Börner, William R. Hazlewood \& Sy-Miaw Lin \\ Indiana University, SLIS \\ 10th Street \& Jordan Avenue, Main Library 019 \\ Bloomington, IN 47405 USA \\ E-mail: \{katy, whazlewo, symlin\} @indiana.edu
}

\begin{abstract}
This paper reports work in progress on the analysis and visualization of the spatial and temporal distribution of user interaction data collected in threedimensional (3-D) virtual worlds. Two tools are introduced. The "WorldMapper" reads in a so-called propdump file and creates a 2-D clickable map showing the layout of the world as well as interaction possibilities such as teleports and clickable web links. The second tool visualizes user interaction data such as navigation, chatting, and Web access activity overlaid on the world map. Resulting visualizations are meant to support social navigation, design evaluation and optimization, and the study of virtual communities. Both tools are demonstrated on a 19-person information treasure hunt for information in a 3-D virtual world. The paper concludes with a discussion and an outlook.
\end{abstract}

Keywords: Information Visualization, Virtual Worlds, Spatio-Temporal Maps, Social Navigation, Usability

\section{Introduction}

The efficient utilization of 3-D virtual worlds (VWs) will require tools that support social navigation, enable the evaluation and optimization of the interface (or "shared space"), and facilitate the study of virtual communities to support their formation and evolution.

Our research aims to demonstrate that advanced data mining and information visualization techniques (Card et al., 1999; Chen, 1999; Ware, 1999; Spence, 2000; Dodge \& Kitchin, 2000) can be advantageously applied to augment, evaluate, optimize, and study collaborative 3-D VWs and to study their evolving communities (Börner, 2001).

In Börner \& Lin (2001), we presented different analysis and visualization techniques that help answer questions such as: How many users participated in a discussion as logged in the chat files? How much overlap exists among the log files? How much do specific users chat, and who chatted the most? How long is the average utterance length for different users? How often do users whisper? How many questions have been asked? Etc.
However, the resulting visualizations gave very limited insight into the spatial and temporal distribution of different user activities. Also, the utterances recorded in the chat log files have been restricted to those from users nearby; i.e., within a limited hearing range.

This paper presents tools for the spatially- and temporally-referenced analysis and visualization of complete sets of user interaction data recorded utilizing bots (software programs).

The next section provides an overview of 3-D world mapping and user interaction visualizations. Section three briefly introduces the 3-D online browser technology used. Section four explains and discusses the WorldMapper tool. Section five introduces different visualizations of user interaction log files, which are used in section six to analyze the data of a 19-person information treasure hunt in a 3-D virtual world. The paper concludes with a discussion and an outlook.

\section{World Maps and Visualizations of User Interaction Data}

Today, computers are highly interconnected via the Internet. A large number of diverse, text -based, 2-D and 3D graphical multi-user systems is available to connect users not only to data but also to expertise of other users. Consequently, there is an increasing interest in the analysis and visualization of social interactions (Donath, 1995; Erickson et al., 1999). See (Börner \& Lin, 2001) for a review of social visualization research.

Another line of research focuses on the visualization of MUDs or 3-D virtual worlds themselves in the form of 2-D or 3-D spatial maps. Martin Dodge's Atlas of Cyberspaces section on MUDs \& Virtual Worlds provides an excellent overview (2000). Elaborated maps by Andrew Smith (Smith, 2000) even show the urban density and the teleport systems of his 3-D world. The AlphaWorld Mapper ${ }^{1}$ by Greg Roelofs and Pieter van der Meulen provides access to a complete, zoomable 2-D map of a VW that has roughly the size of California $\left(429,025 \mathrm{~km}^{2}\right)$.

\footnotetext{
1 http://mapper.activeworlds.com/aw/
} 
The existing systems teach many valuable lessons about how to visualize online spaces, their users, navigation and conversation activity, as well as "social" relationships. However, the analysis of computer-mediated communication is dominated by textual analysis without spatial reference, using linguistic discourse analysis and ethnographic methods (Herring, 1996). Studies that examine graphical chat rooms (e.g., Suler's Palace study) have used field observations, e-mail interviews, studies of mailing lists and participant observation exclusively. Naper (2000) was among the first to analyze chat text logged in a 3-D VW and calls for analysis of graphical elements.

To our knowledge, there exists no system that traces and visualizes spatially- and temporally-referenced user interaction such as navigation, object manipulation, Web access, or chatting in 3-D VWs. This may be due to the fact that most $3 \mathrm{D}$ online browser systems provide an exclusive chat log (oftentimes restricted to a fixed number of close-by avatars) of a 3-D world experience. Information about when and where a certain utterance was made, user positions, object movements, teleports/warp usage, or Web accesses is lost. However, spatial references are vital to identify places where people typically speak, to find teleport designs that work, etc. and ultimately to augment, evaluate, optimize, and study 3-D VWs and their evolving communities (Börner \& Lin, 2001). Temporal aspects of user interactions are key to identify user-user interaction and collaboration among others.

This paper presents and demonstrates two tools that aim to benefit the design, evaluation, optimization, and study of 3-D VWs and their communities.

\section{Three-Dimensional Online Browser}

Today, a large variety of commercial online browser systems can be used to design customized 3-D VWs. Among the most frequently used systems are Blaxxun's online community client-server architecture, ${ }^{2}$ Microsoft's Virtual Worlds Platform, ${ }^{3}$ Active Worlds technology by Activeworlds, Inc., ${ }^{4}$ and the new Adobe Atmosphere browser. 5 Damer (1998) and Börner (2001b) provide reviews of different browser systems and existing virtual communities.

Available 3-D browsers vary in many details, such as their programming language, storage methods for objects, and how 3-D objects are rendered for 2-D display. However, all the systems mentioned above facilitate the

\footnotetext{
2 http://www.blaxxun.com/community

3 http://www.vworlds.org/

4 http://www.activeworlds.com/

5 http://www.adobe.com/products/atmosphere/
}

creation of multi-modal, multi-user, navigable, and collaborative virtual worlds in 3-D that are interconnected with standard Web pages and are accessible from standard computer platforms via the Internet, 24 hours a day and 7 days a week. Mouse, keyboard, and screen are used as the main human computer interface, as are commonly used interactions such as point and click, icons, and menus. However, the browsers provide access to a 3-D virtual world that users can enter and explore together.

Browser systems differ in the ease of building within the worlds, their scalability and extensibility, and the size of the user communities they manage to attract.

Active Worlds (AW) stands out due to its exceptionally fast browser download, easy installation, and surprisingly low system requirements. AW is based on Render Ware, an interactive 3-D Graphics API. It differs from $V R M L$-based systems in the ease with which participants can build within the world. A user simply selects an existing object, makes a copy of it, and renames it as a different object. A large object library is available. The real-time object download is based on proximity and enables the creation of very large worlds. AW has a large user community dating back to mid-90s. As one of the most popular VW systems, it hosts over six thousand different worlds in its main entertainment-oriented universe, and more than one hundred sixty worlds in EduVerse, a special universe with an educational focus.

Figure 1 shows the AW interface. In contains three main windows: a 3-D graphics window populated by avatars, a Web browser window, and a chat window. At the top are a menu bar and a toolbar for avatar actions. Users can collaboratively navigate in $3 \mathrm{D}$, move their mouse pointer over an object to bring up its description, click on 3-D objects to display the corresponding Web page in the right Web frame of the AW browser, or teleport to a different area. The browser maintains a history of visited places and Web pages so that the user can easily return to previous locations and pages.

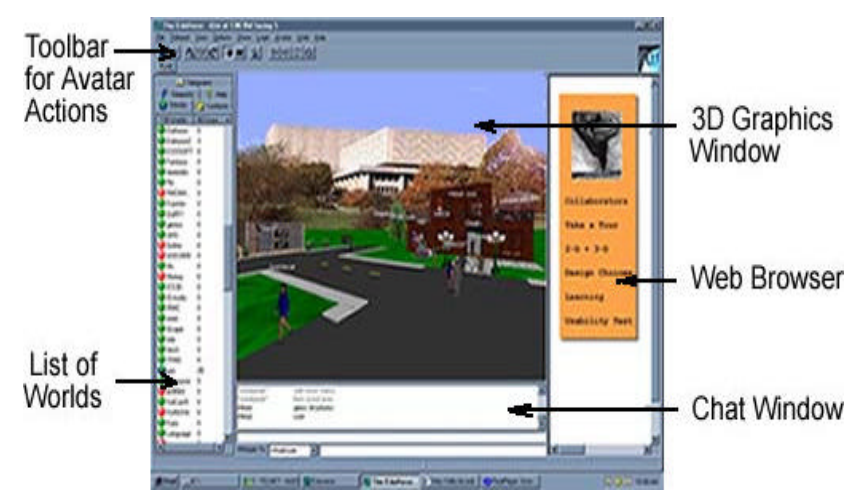

Figure 1. The AW browser interface 
The Information Universe ${ }^{6}$ (iUni) (Katy Börner, 2001a) is a world in AWs EduVerse. An overview map of this $400 \mathrm{~m}$ by $400 \mathrm{~m}$ world rendered in $2-\mathrm{D}$ based on the list of objects that made up the world on Feb 22 2002 is displayed in Fig. 2. All 3-D objects are rendered in white. Web links and teleports are indicated by green and red dots respectively. This map is clickable and was generated by the WorldMapper explained in section four.

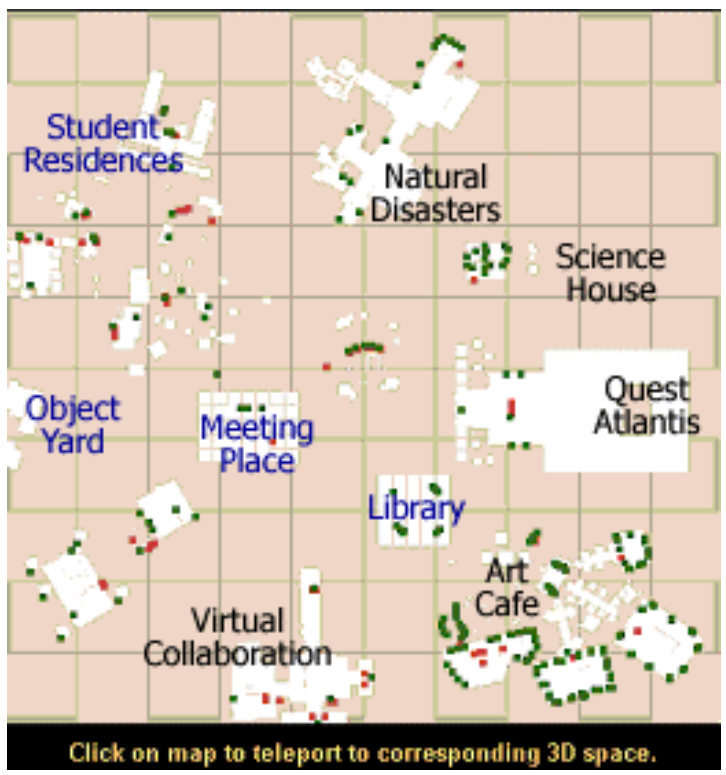

Figure 2. Map of iUni with Web links (green) and teleports (brown). Major areas are labeled.

There are five different learning environments - a Natural Disaster Area, a Science House, a Quest Atlantis portal to different theme parks for kids, an Art Cafe, and a Virtual Collaboration area. Black and blue area labels denoting the five learning environments and community areas respectively have been added by hand.

\section{WorldMapper}

World mapping comprises data collection, analysis, visualization and interaction design.

Data Collection. In AW, information on objects that make up a virtual world - such as time of building, builder, position, orientation, object name, its description (displayed when user moved mouse pointer over this object), and any actions such as teleporting or display of web links - are stored in the object file also called propdump file), and can be obtained by the owner of a world.

\footnotetext{
${ }^{6} \underline{\text { http://ella.slis.indiana.edu/ katy/iUni/ }}$
}

The dimensions of each object - used to determine and avoid encroachment during object design and manipulation - are specified in the so-called registry file. ${ }^{7}$

Data Analysis. In order to render a map, the object file is parsed for the complete set of objects, their positions, and rotations. The exact dimensions of each object are determined by consulting the registry file. In addition, teleports and web links are identified and stored separately. Subsequently, object positions and sizes, as well as positions of teleports and web links, are scaled according to the size of the requested map.

Visualization and Interaction Design. The visualization consists of a grid of reference, objects, marks for teleports and web links, and a legend that are drawn in this sequence. The map is clickable; i.e., a mouse click on a certain area on the map instantaneously teleports the user into the corresponding area in the 3-D VW, easing navigation.

The Active WorldMapper interface is available online at http://iuniverse.slis.indiana.edu/map/. Users can enter general information such as world name, size, and contact person information as well as an object file and registry file (optional). A click on "Map it" results in the rendering of a two-dimensional, clickable map like the one shown in Fig. 2.

\section{User Visualization}

The visualization of user interaction data requires the collection of usage data, data analysis, and data visualization.

Note that we are using Active Worlds technology. Other browser systems may require different user logging and visualization approaches.

Data Collection. In AW, the hearing range is limited to, maximally, a $120 \mathrm{~m}$ distance. Accordingly, chat log files only collect utterances of users' avatars that are in close proximity.

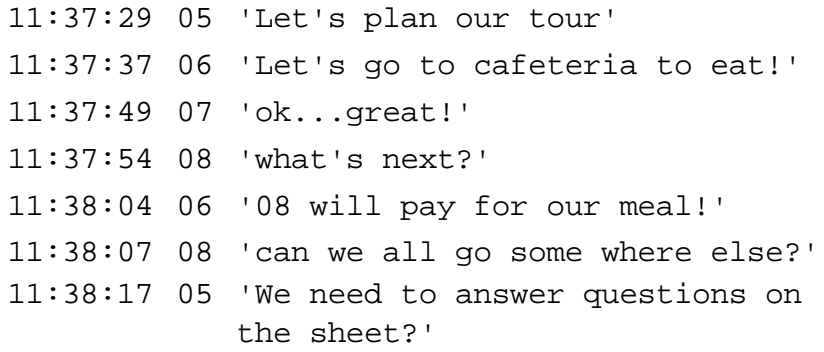

Figure 3. Time-stamped chat utterances

7 A sample AW registry file is available online at http://www.activeworlds.com/help/registry.html. 
To ensure complete chat logs, a program named LogBot was utilized. This program logs all user positions. Chat and navigation actions are time-stamped. Figure 3 shows a section of the time-stamped chat utterances of subjects 05 through 08 that acted as members of team 2 in the treasure hunt (see section six).

Data Analysis \& Visualization. Time-stamped chat and position data can be utilized to visualize spatially and temporally referenced navigation and chat activity of users. Diverse visualizations are discussed in section six.

\section{Information Treasure Hunt}

The proposed visualization tools have been applied to analyze and visualize data collected during an "Information Treasure Hunt" in a 3-D VW.

The study took place in a laboratory setting using PC computers with 19-inch monitors. Nineteen subjects - ten males and nine females - participated. Subject completed degrees varied with nine at graduate-level coursework and ten at undergraduate-level coursework. Their backgrounds included Information Science (4), Instructional Systems Technology (3), MLS/MIS (1), Leisure Studies (1), Computer Science (8), Electronics \& Communication Engineer (1), and other (1). Subjects' average age was 28. Three of the subjects were native speakers in English, eight in Mandarin Chinese, three in Korean, three in Hindi, one in Tamil and one in unspecified language. Eighteen of the subjects were right-handed, and only one is lefthanded. Seventeen of the subjects touch-type. Their average number of hours spent on the computer per week is 34.11 hours and the average weekly hours on the Internet is 24.11 hours. Three of the subjects rated their knowledge on computers as 5 (5 being expert), eleven rated themselves as 4 , four as 3 , and one as 2 . Seventeen of the subjects had visited a text -based chat environment, seven had visited a 3-D single user virtual environment, and four had visited a 3-D multi-user virtual environment before.

Subjects in this study were instructed to login to "iUni" by starting the EduVerse Browser from the desktop. Subjects were provided with a brief introduction to the environment and navigational instructions and had five minutes to explore the different navigation and interaction possibilities.

The main task was to collectively search for the answers to ten questions (Q1-Q10) about the world comprising:

1. How many student residences are there?

2. Write down the names of all students that have residences.

3. Find 'Magster's Mansion' and answer: How many worlds are linked from her menu system?
4. In the 'Meeting Place' area you will find a sign that links to lecture slides. What was the topic of this particular lecture?

5. What are the four main resources that are available in the 'Library'?

6. How many different areas are in the 'Art Café'?

7. In the 'Quest Atlantis' area: Is 'Jonassan' displayed in the 'Tower of Power'? yes/no

8. How many household appliances are linked from the 'Science House'?

9. In the 'Natural Disaster' area: How many different types of buildings are dis played?

10. How many video streams are accessible in the 'Virtual Collaboration' area?

The answers had to be reported by one team member. To complete the task, subjects needed to find their assigned teams and decide on a strategy to quickly find information necessary for answering the provided questions. Also, teams had to agree on how to exchange information with each other. Figure 4 shows the locations of answers to Q1Q10.

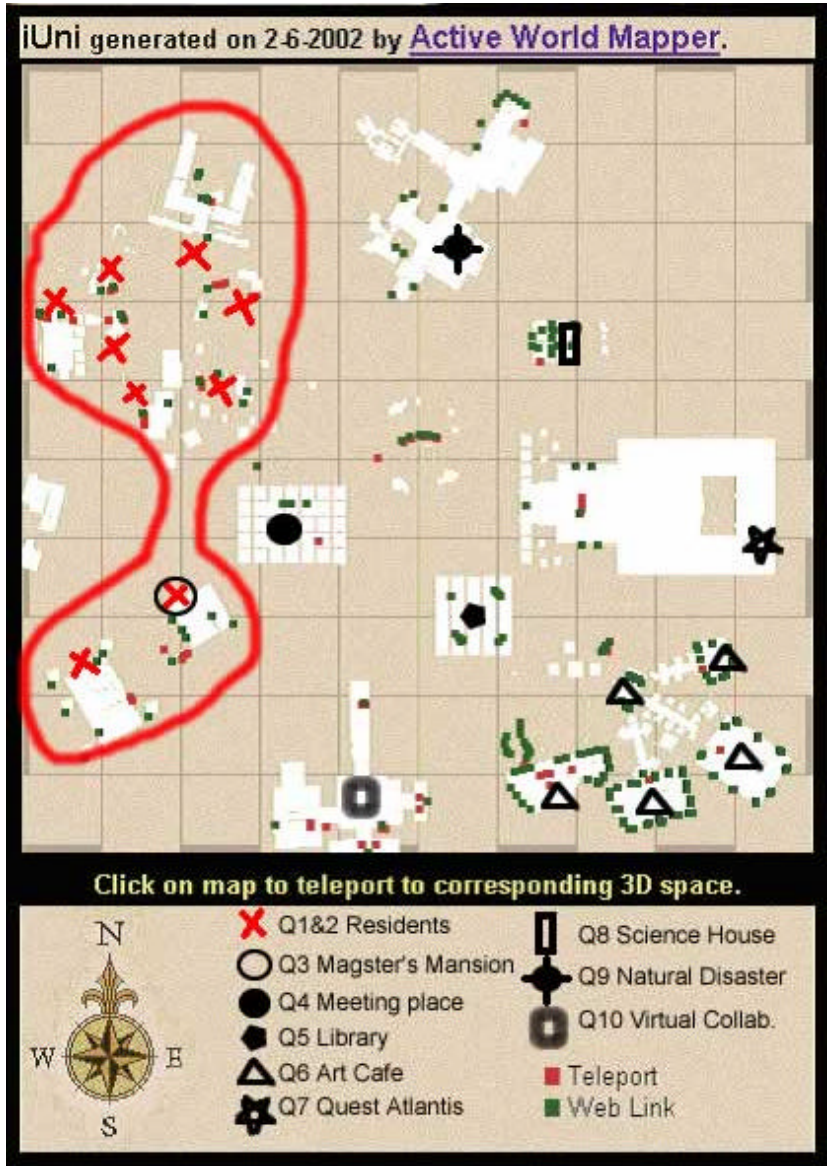

Figure 4. Places that provide answers to Q1-Q10 
Subjects did have access to and used a static, nonclickable map such as the one displayed in Fig. 2 for navigation.

Figure 5 visualizes the number and length of utterances made by the $19 \mathrm{~S}$ 's during the information treasure hunt. Subject numbers are plotted on the xaxis. The y-axis represents the number of utterances. Each utterance is represented by a rectangle of uniform height. The width of the rectangle corresponds to the number of more or less meaningful "words" in the utterance. Utterances containing more than 14 words are displayed in red. Shorter messages are shown in light yellow. Subject number 09 clearly posted most messages - exactly 101 . While 01 posted only 11 (although rather long) messages.

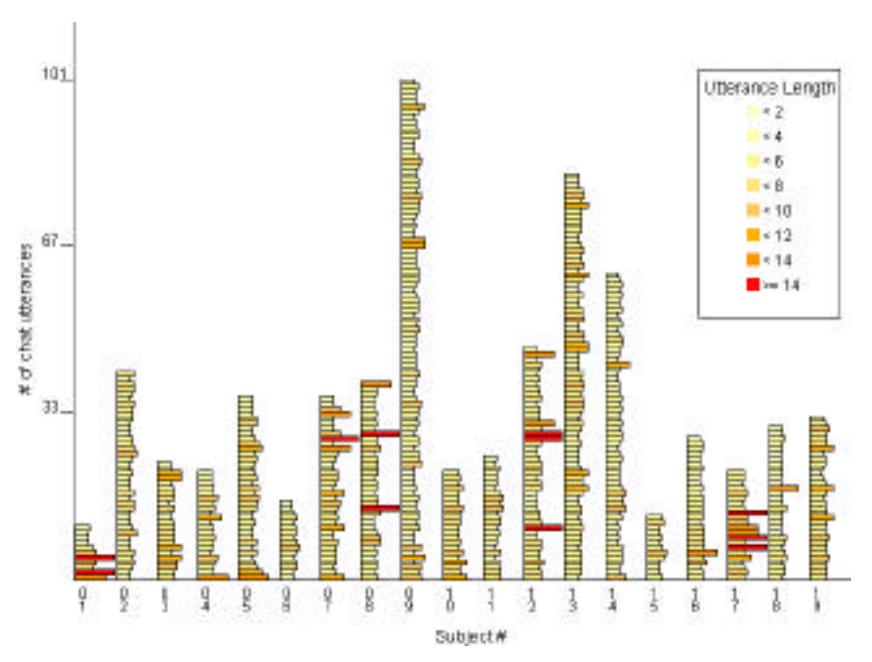

Figure 5: Number and length of utterances

Figure 6 shows the chat (yellow) and web access (red) activity for subjects 01-19 over the entire time period. Teams are labeled in blue.

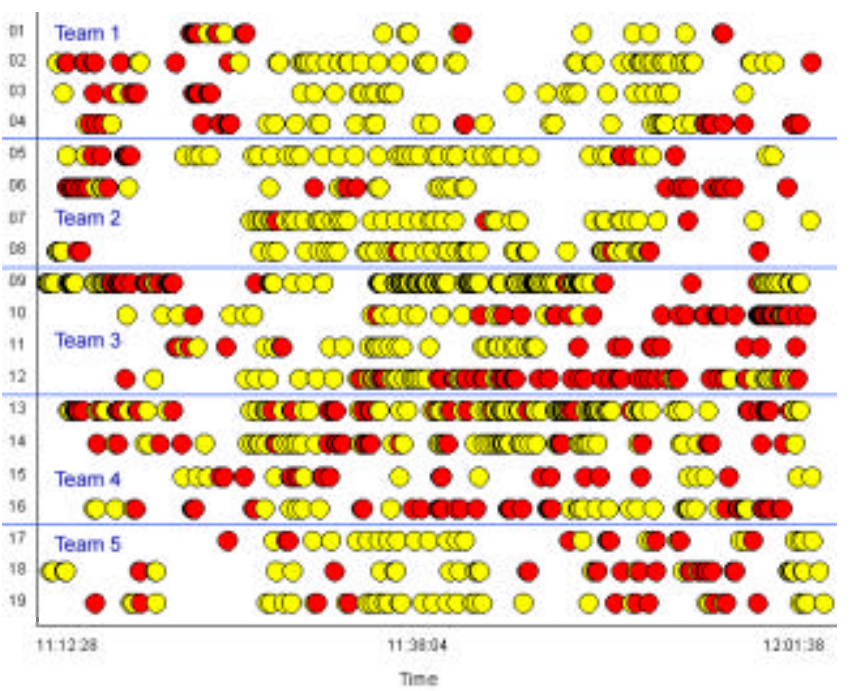

Figure 6. Time sequence of chat activity
The first chat utterance was made around 11:12a. The first chat-based attempt to find team members was made at 11:24:22 when subject no 10 said 'hi 15 i am looking for you'. The time in-between is filled with the exploration the browser interface, greetings, and first interactions with the VW. Its follows a very active time in which Ss try to find all their team members. According to the chat $\log$, team 4 succeeds first at 11:32:40. Subsequently, teams get a chance to concentrate on answering the ten questions. No team finishes this task in the given time but several teams manage to exchange some answers at the end. Accordingly, Fig 6 shows times of active chat interaction and solitary information search.

Figure 7 shows the navigation trails of team 2 (subjects 05-08), team 3 (subjects 09-12), and team 4 (subjects 13-16) plotted after 15, 30, 45 and 60 mins. Analyzed in combination with the detailed chat logs, team 2 and 3 divided the questions and exchanged the answers they obtained at the end. Team 4 decided on a simple protocol, such as MOV for moving and HALT for halting.

All three teams have been coordinated and busy searching for answers and exchanging information in the last 15 mins. With regard to the highest scores the teams gained, team 2 has the most answers, and team 4 has the least. The strategy that team 4 employed took them way too much time in negotiating. A spatial comparison of the navigation trails with Fig. 4 reveals which of the answers could have been found by the different teams.

The analysis of the pre-test questionnaires revealed information on different search strategies such as consulting a 2-D map of the world (similar to figure 1), flying up to locate team members, view the main places to find out key words of the target, and chat. Chat was reported as the main information exchange strategy by 15 S's. Seven Ss reported that they tried to meet with their team members to divide the tasks and then go to different places to find answers. Among the problems encountered by subjects are: $6 \mathrm{~S}$ 's reported difficulty in communicating with others (possibly due to the limited hearing range), 6 $\mathrm{S}$ 's reported getting lost in iUni, and one reported losing sense of direction. Also, we asked S's for what purpose they used the 3-D world and chat. Result for 3-D world consisted of the following: for fun (6), navigation (6), locate answers (5), meet teammates (1), learning (1). Chat was used for: idea exchange (8), finding teammates (5), communication (5), recognizing \& acknowledging signals (1), finding locations (1).

Asked for other comments, S's acknowledged good interface design. Although some got lost multiple times, they reported they felt very excited. Three S's found it very interesting. Two mentioned again that it is easy to lose sense of direction, and hard to contact team members. 

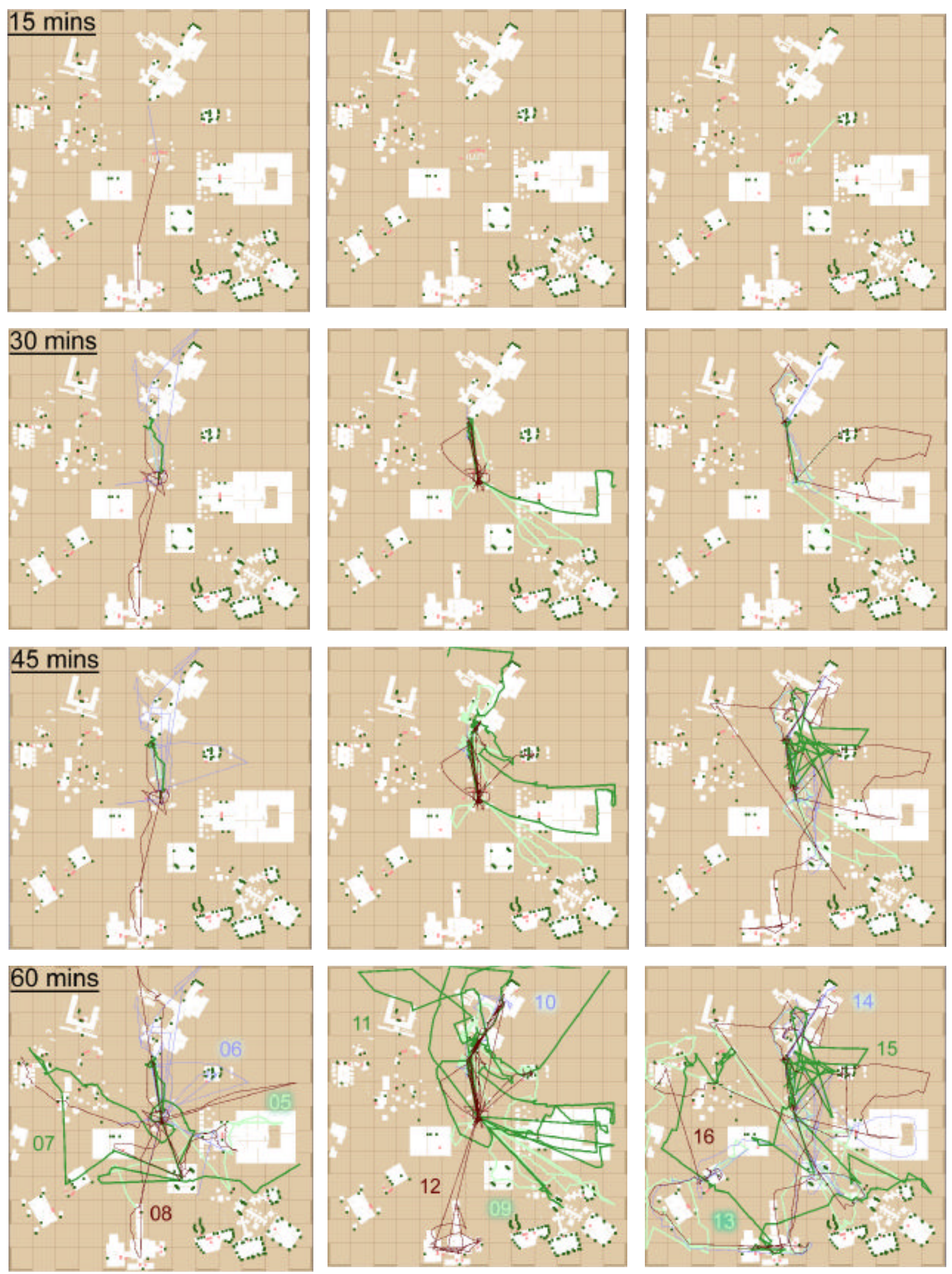

Figure 7. Spatial navigation trails of team 2 (left), team 3 (middle) and team 4 (right) 
Suggested improvements are to add a "private chat room" for each team (1); and a more meaningful map should depict position; should display the direction like "north" or "up" (2); and to give more time to find answers (1).

\section{Discussion \& Outlook}

The paper presented an analysis and visualization of user interaction data that reveals the spatial and temporal distribution of different user interactions in a 3-D VW.

The visualizations are intended to assist users in making sense of the world, its information resources, and collaboration possibilities, to aid designers with the organization and layout of world content and the selection of interaction possibilities, and to enable researchers to study the communities evolving in 3-D VWs.

Future work aims at a real-time visualization of user positions to be displayed in a clickable map to support social navigation. This will require the continuous collection of usage data, data analysis, and data visualization. We will test this map by re-running the very same Treasure Hunt experiment. It is expected that the map will ease finding other team members as well as spatial navigation.

While demonstrated and tested in a simple Information Treasure Hunt, it should be obvious that the proposed mapping and user interaction visualization tools are applicable to support diverse applications, e.g., education, e-commerce, or entertainment, that utilize multi-user VWs.

Given the ease in monitoring almost all activity in the virtual space, care has to be taken to ensure that extensive user tracking and analysis benefit the users rather than invading their privacy. At any point in time users need to be fully aware what interaction data is being recorded, analyzed, and visualized. Otherwise, the system is likely to approach the status of a panopticon.

\section{Acknowledgements}

Several students are involved in this research including Alan Lin, Hakan Tuzun, and Min Xiao. Martin Dodge provided insightful comments on an earlier version of this paper.

We acknowledge ActiveWorld's generous support in providing free hosting of 3-D VWs for educational purposes as well as an active research environment in EduVerse. The research was supported by a High Performance Network Application grant from Indiana University 2000/2001.

\section{References}

Börner, K. (2001, October 18-20). Twin Worlds: Augmenting, Evaluating, and Studying ThreeDimensional Digital Cities and Their Evolving
Communities (to be published by Springer). Paper presented at the The Second Kyoto Meeting on Digital Cities Digital Cities 2001 - International Symposium and Workshop, Kyoto, Japan,

Börner, K. (2001a). Using Active Worlds Technology to build an iUniverse of 3-D collaborative learning environments. IEEE Learning Technology newsletter. Available: http://lttf.ieee.org/learn tech/issues/january2001/. Börner, K. (2001b). Virtual World Technologies for Digital Cities of Tomorrow. Invited Talk at the Panel Collaboration in Building Future Digital Cities. The Second Kyoto Meeting on Digital Cities, Kyoto, Japan. Available: http://ella.slis.indiana.edu/ katy/research/dc01panel.pdf.

Börner, K., \& Lin, Y.-C. (2001, July 25-27). Visualizing Chat Log Data Collected in 3-D Virtual Worlds. Paper presented at the Information Visualisation Conference, London, England: IEEE Press, pp. 141146.

Damer, B. (1998). Avatars! Exploring and Building Virtual Worlds on the Internet. Berkeley, CA: Peachpit Press.

Dodge, M. (2000). An Atlas of Cyberspaces. Multi-User Dimensions (MUDs) \& Virtual Worlds. Available: http://www.geog.ucl.ac.uk/casa/martin/atlas/muds v w.html.

Donath, J. S. (1995, November 5-9, 1995). Visual Who: Animating the affinities and activities of an electronic community. Paper presented at the ACM Multimedia 95, San Francisco, California: ACM Press, Erickson, T., Smith, D. N., Kellogg, W. A., Laff, M., Richards, J. T., \& Bradner, E. (1999). Socially Translucent Systems: Social Proxies, Persistent Conversation, and the Design of "Babble". Paper presented at the Proceeding of the $\mathrm{CHI} 99$ Conference on Human Factors in Computing Systems: The CHI is the limit: ACM Press, pp. 72 - 79.

Herring, S. C. (Ed.). (1996). Computer mediated communication. Linguistic, social and cross-cultural perspectives. Amsterdam/Philadelphia: John Benjamins Pub Co.

Naper, I. (2000). System features of an inhabited 3-D virtual environment supporting multimodality in communication. Paper presented at the 34th Annual Hawaii International Conference on Systems Sciences: Los Alamitos, CA: Institute of Electrical and Electronics Engineers (IEEE) Computer Society.,

Smith, A. (2000). Mapping 30 days. CASA. Available: http://www.casa.ucl.ac.uk/30days/mapping.htm 